\title{
Re: Post-renal Transplant Urolithiasis in Children: An Increasingly Diagnosed Complication: A Retrospective Cohort Study
}

\author{
Sophia Ma1,2, Amir Taher1, Benjamin Zhu11, Anne Maria Durkan1,3 \\ 1 Westmead Children's Hospital Clinic of Nephrology, New South Wales, Australia \\ 2 New South Wales University Faculty of Medicine, Sydney, Australia \\ 3Sydney University Faculty of Medicine, Department of Paediatrics, Sydney, Australia
}

Arch Dis Child. 2020;105(1):69-73. Doi: 10.1136/archdischild-2019-317203

\section{EDITORIAL COMMENT}

The prevalence of kidney stones in the general population is increasing not only in adults but also in the pediatric population with a greater frequency in girls compared with boys. Urolithiasis is an uncommon complication, usually presenting with unusual findings in kidney transplant recipients. However, if left untreated, it can be a potential cause for graft loss. In this retrospective cohort study, the investigators evaluated the frequency, risk factors and characteristics of post kidney transplantation (KTX) urolithiasis in 142 pediatric kidney transplant recipients over two decades (1995-2016) in an Australian hospital. Only 8 of 142 (5.6\%) male recipients were diagnosed with stones; the rate is higher than the previously reported (2.7\%-5\%) in the early 2000s. Interestingly, stone formers were significantly younger at the time of transplantation and weighed substantially less than non-stone formers. Time to presentation was bimodal; three stones were diagnosed in the first 3 months after $\mathrm{KTX}$, whereas the initial postoperative ultrasound studies were negative for urolithiasis; retained sutures and recurrent urinary tract infections were the most likely causes for early occurrences. The remainder stones were identified 2-4 years after KTx. All stones contained calcium oxalate, 3 were mixed with calcium phosphate and there were only one uric acid stone. Although hypercalciuria was present in 5 (83.3\%), none had hyperuricosuria and hypercalcemia. Graft loss due to urolithiasis was not experienced in this cohort. Besides being a single-center study, its relatively small sample size and retrospective characteristics make it hard to make some strong comments regarding the prevalence of childhood urolithiasis and biochemical risk factors in pediatric KTx population, however, there is an increase in the frequency of urolithiasis in parallel with the increase in the general pediatric population.

Yarkın Kamil Yakupoğlu, MD

${ }^{\circ}$ Copyright 2020 by the Association of Urological Surgery / Journal of Urological Surgery published by Galenos Publishing House. 